\title{
T-ray biosensing: a versatile tool for studying low-frequency intermolecular vibrations
}

\author{
Bernd M. Fischer ${ }^{a, b}$, Morten Franz ${ }^{a, b}$, and Derek Abbott ${ }^{a}$ \\ ${ }^{a}$ Centre for biomedical Engineering and School of Electrical \& Electronic Engineering, The \\ University of Adelaide, Adelaide SA, 5005, Australia; \\ ${ }^{b}$ Department of Molecular and Optical Physics, Albert-Ludwigs-Universität Freiburg, D-79104 \\ Freiburg, Germany
}

\begin{abstract}
The terahertz (or T-ray) spectra of many small molecules of biological relevance show very characteristic, specific features that are sensitive to small changes of the molecular structure and even isomerization. On the other hand, most packaging materials like plastics, paper or even clothing are transparent for T-rays. Therefore, it is possible to differentiate and identify different substances by their spectral fingerprints, even through their packaging. This supports the potential of this technique in a wide range of applications from safety and security applications, via biosensing, through to pharmaceutical quality control.

However, most of the molecular vibrations that give rise to the characteristic features in the T-ray spectra are phonon-like intermolecular vibrations of weakly bound crystalline compounds. This can be easily demonstrated by comparing the spectra of different crystals of the same molecule. Whereas this sensitivity on the intermolecular structure can be used to probe the crystalline structure and detect phase transitions, it is a hurdle when it comes to identify samples that lack such a well defined intermolecular structure. Yet, we have recently shown that a comparison of the absolute absorption values can still be used to differentiate between complex biomolecules such as RNA.

In this paper we will demonstrate, based on a wide range of spectra, the potential of T-ray spectroscopy for biosensing and will show examples where this technique can be used to probe the crystalline configuration and probe phase transitions and will discuss the feasibility of using this technique for biosensing.
\end{abstract}

Keywords: Terahertz time-domain spectroscopy, hydrogen-bonds, biomolecules, intermolecular vibrations, T-rays

\section{INTRODUCTION}

Although first FIR spectra of biomolecules were reported in the early seventies of the previous century, ${ }^{1,2}$ the recording of spectra at such low frequencies was very difficult. It was only after the introduction of $\mathrm{THz}$ time-domain spectroscopy ${ }^{3,4}$ that this frequency range became more accessible for the spectroscopic study of molecules of biological interest. First T-ray measurements focussed on either large molecules such as DNA or small molecules like for example retinal. ${ }^{6}$ In particular the spectra of small molecules ${ }^{7-9}$ typically show several distinct, characteristic features, and are highly sensitive to small changes of the molecular structure or even isomerization. In reference to these very characteristic spectral signatures, the far-infrared part of the electromagnetic spectrum is often referred to as fingerprint region. As most non-polar and non-liquid materials are transparent for far-infrared radiation, this sensitivity is of pronounced interest for non-contact identification of samples through packing material via their spectral signatures.

It as been shown recently ${ }^{9-12}$ that many of the characteristic $\mathrm{THz}$ signatures originate from intermolecular vibrations of the weakly bound crystalline lattice rather than from intramolecular vibrations, like for example

Further author information: (Send correspondence to Bernd M. Fischer)

Bernd M. Fischer: E-mail: bernd.fischer@adelaide.edu.au, Telephone: +61 883034115

Morten Franz: E-mail: morten@cip.physik.uni-freiburg.de, Telephone +49 7612035871

Derek Abbott: E-mail:dabbott@elecengadelaide.edu.au, Telephone: +61 883035748

Biomedical Applications of Micro- and Nanoengineering III, edited by Dan V. Nicolau, Proc. of SPIE Vol. 6416, 64160U, (2006) · 1605-7422/06/\$15 - doi: 10.1117/12.695726 
torsional vibration or phonon-like vibrations of long-chained molecules. Therefore, the $\mathrm{THz}$ spectra of many larger, more complex molecules that lack a well defined crystalline structure, show only few or no distinct, characteristic features. In this paper we will show several examples to demonstrate the intermolecular character of the lowest modes in the far-infrared spectrum of many biomolecules and will discuss the possibility of exploiting this sensitivity to different crystalline structures for study of weak intermolecular binding structures in these samples and to probe crystallinity.

\section{EXPERIMENTAL}

The THz spectra shown in this work have been recorded with three different systems in Germany and Australia: Most of the spectra were recorded at the University of Freiburg/Germay using two custom built THz timedomain spectrometers. One was operating in transmission geometry, whereas the other was operating in reflection geometry. The third spectrometer used in this work is a commercially available fibre-coupled $\mathrm{THz}$ spectrometer (T-ray 2000, Picometrix) and is located at the National T-ray Facility in Adelaide.

All THz time-domain spectrometers used in this study are based on photoconductive antennas in order to generate and detect the $\mathrm{THz}$ pulses. By illuminating the gap between the two metal lines the resulting free carriers are accelerated in the bias field and thus lead to the emission of a short electromagnetic pulse. The antennas of the custom built systems in Freiburg are made of LT grown GaAs (Freiberger Compound Materials $\mathrm{GmbH})$. The antenna geometry consists of two photolithographically deposited parallel metal ( $\mathrm{Au} / \mathrm{Ge} / \mathrm{Ni})$ striplines, separated by several tens of micrometers. The detector chip consists of a similar structure, yet no bias is applied on the metal lines. Only when the incoming $\mathrm{THz}$ pulse and the electromagnetic pulse temporally coincide at the detector, a current proportional to the field strength of the $\mathrm{THz}$ pulse can be detected. By varying the time delay between the $\mathrm{THz}$ pulse and gating femtosecond laser pulse, the full temporal shape of the $\mathrm{THz}$ pulse can be recorded. The dielectric function of a material is determined in transmission THz-TDS by measuring a reference pulse $E_{\text {ref }}(t)$ propagating through an empty spectrometer and a sample pulse $E_{\text {sam }}(t)$ propagating through the spectrometer with the sample of thickness $d$ placed in the beam path. The field of the THz pulse transmitted through the sample $E_{\mathrm{sam}(\mathrm{t})}$ is modified by the dispersion $n(\nu)$ and absorption $\alpha(\nu)$ of the sample. The two pulses are transformed into the frequency domain, and the amplitude $A(\nu)$ and phase $\Phi(\nu)$ of the ratio of the two spectra,

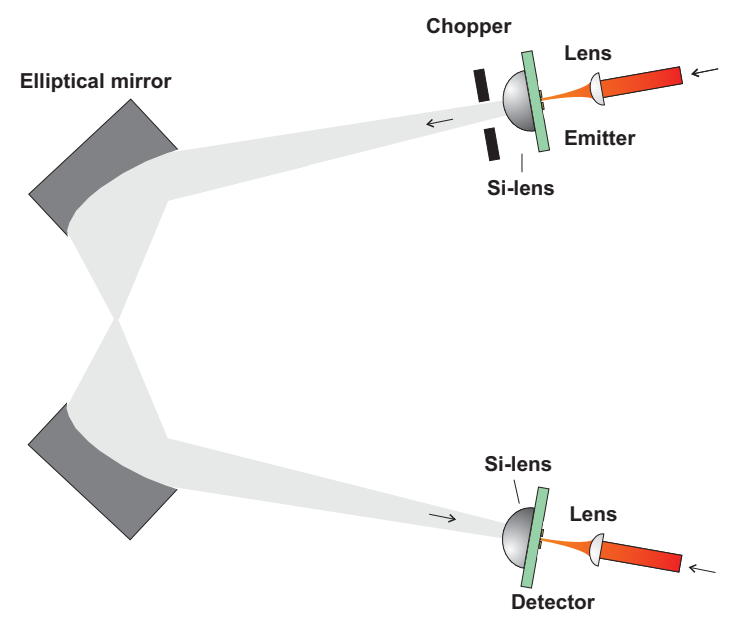

(a)

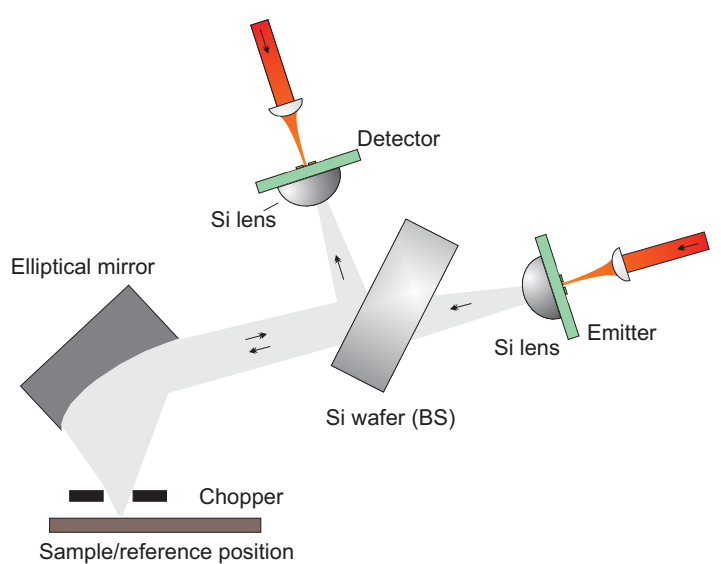

(b)

Figure 1. Schematic diagram of the custom built $\mathrm{THz}$ time-domain spectrometer in (a) transmission geometry and (b) in reflection geometry. 

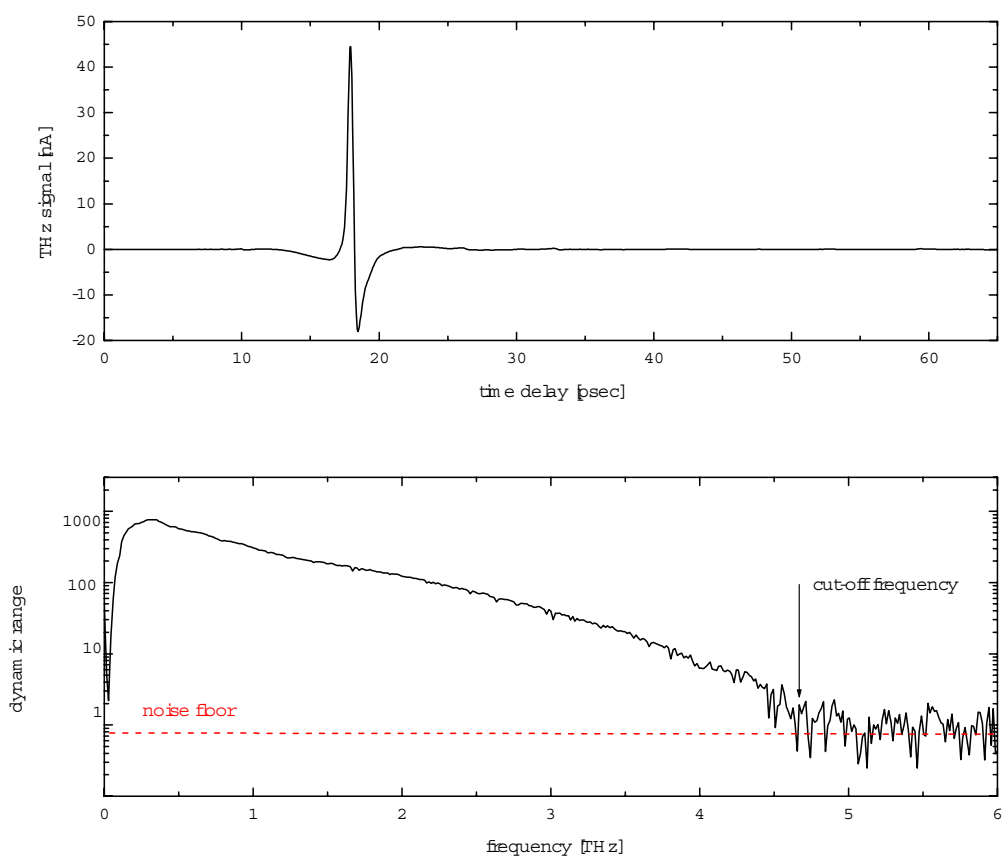

Figure 2. Typical $\mathrm{THz}$ pulse (upper curve) and corresponding frequency spectrum of the custom-built transmission time-domain spectrometer in Freiburg/Germany. The upper limit of the accessible frequency range is reached when the detected signal reaches that of the noise floor as indicated in the lower graph.

$$
\begin{aligned}
\frac{E_{\mathrm{sam}}(\nu)}{E_{\mathrm{ref}}(\nu)} & =\frac{4 n}{(n+1)^{2}} \exp [-\alpha d / 2+i 2 \pi \nu(n-1) d / c] \\
& =A(\nu) \exp [i \Phi(\nu)]
\end{aligned}
$$

are calculated and analyzed to obtain the spectra of the absorption coefficient

$$
\alpha(\nu)=-\frac{2}{d} \ln \left\{A(\nu) \frac{[n(\nu)+1]^{2}}{4 n(\nu)}\right\}
$$

and index of refraction

$$
n(\nu)=1+\frac{c}{2 \pi \nu d} \Phi(\nu) .
$$

In Figure 2, a typical $\mathrm{THz}$ pulse is shown together with its frequency spectrum. Owing to the typical singlecycle nature of the $\mathrm{THz}$ pulse, its frequency spectrum extends from the low gigahertz region to several THz. At high frequencies the spectrum is characterized by a gradual roll-off, until the detected signal level approaches that of the noise floor of the experiment.

For some samples a measurement in transmission geometry is not applicable. In particular water and other polar solvents show a very strong absorption in the far-infrared region. Also phonon-modes in ionic crystals show typically absorptions that exceed the limits given by the dynamic range of the experiment. ${ }^{13} \mathrm{~A}$ method to circumvent the limitations of THz-TDS, described above, is to operate the $\mathrm{THz}$ spectrometer in the reflection mode. In this case the reference signal typically consists of the $\mathrm{THz}$ pulse reflected from a silver mirror surface 
with a reflection coefficient close to minus unity. The sample signal consists of the $\mathrm{THz}$ pulse reflected from the plane sample surface, positioned at the same plane as the reference surface. Alternatively, the reference and sample signals are obtained as reflections from the front and the backside of a thick window in front of the sample. ${ }^{14}$ Here we consider only the case of normal incidence with a metal mirror as a reference surface. In the frequency domain the ratio between the sample and the reference spectra is then

$$
A(\nu) \exp [i \Phi(\nu)]=\frac{\hat{n}-1}{\hat{n}+1}=\frac{n+i \kappa-1}{n+i \kappa+1} .
$$

Equation 4 can be inverted, and simple expressions for the index of refraction and absorption coefficient can be found:

$$
\begin{gathered}
n(\nu)=\frac{1-A(\nu)^{2}}{1+A(\nu)^{2}-2 A(\nu) \cos \Phi(\nu)}, \\
\alpha(\nu)=\frac{2 \pi \nu}{c} \frac{2 A(\nu) \sin \Phi(\nu)}{1+A(\nu)^{2}-2 A(\nu) \cos \Phi(\nu)} .
\end{gathered}
$$

In order to record the temperature dependence of the spectral features, the samples are mounted in a cryostat equipped with TPX windows. The temperature is measured near the sample by a calibrated Si-diode with an accuracy of $\pm 1 \mathrm{~K}$. The cryostat can be moved so that the $\mathrm{THz}$ beam passes through either the sample or through an empty aperture of the identical size as the clear aperture of the sample. By mounting the sample on a XYstage, a two dimensional image of the sample can be recorded, where each pixel consists of the full spectral information.

\section{FAR-INFRARED SPECTRA OF POLY- AND SINGLE-CRYSTALLINE SAMPLES}

The far-infrared spectra of various molecules of biological interest show distinct, characteristic features. These spectroscopic signatures are very sensitive to the molecular structure or even isomerization, ${ }^{6}$ and it is possible to identify a specific isomer by its far-infrared fingerprints - however, very little is known about the low-frequency vibrations that give rise to these signatures. Due to the delocalized, global character of these vibrations, the environment and neighboring molecules have to be taken into consideration, and therefore an assignment of the modes via comparison of the experimental data with calculated spectra based on DFT techniques is in most cases not applicable - as the large number of atoms in the system that have to be considered make such calculations, within a reasonable CPU usage time, impossible. However, it has recently been shown in a few cases that the vibrations that give rise to the features below $200 \mathrm{~cm}^{-1}$ are typically intermolecular vibrations of weakly bound crystalline structures. ${ }^{9-12}$

By comparing the spectra of different similar molecules, it is possible to directly demonstrate the intermolecular character of the low frequency vibrations that give rise to the specific $\mathrm{THz}$ signatures. Previously, we reported the far-infrared spectra of amorphous and polycrystalline sucrose ${ }^{7}$ as well as $\alpha$-D-glucose and $\alpha$-glucose monohydrate. ${ }^{9}$ It was shown that all distinct features appearing in the terahertz spectrum of polycrystalline sucrose are not observed in the amorphous sample. In the case of anhydrous and monohydrated glucose, the terahertz spectra showed very different features, despite the fact that both samples contain $\alpha$-D-glucose molecules in a very similar configuration. The only difference lies in the intermolecular binding parameters of three samples, i.e. the additional water molecule incorporated in the crystalline structure of the monohydrated sample. This indicates that the observed spectral signatures originate rather from intermolecular vibrations than pure intramolecular vibrations.

\subsection{Enantiopure and racemic crystals}

Another class of samples that are particularly suited to demonstrate the intermolecular character of the farinfrared vibrations are racemic crystals. Enantiomers are Stereoisomers (i.e. isomers that have the same bond configuration) that only differ from each other in the spatial arrangement of the atoms and functional groups in such a way that the resulting molecules are mirror images of each other. The naming convention is either based on the ability of this solution to turn the polarization vector of light as it passes through a solution of these 


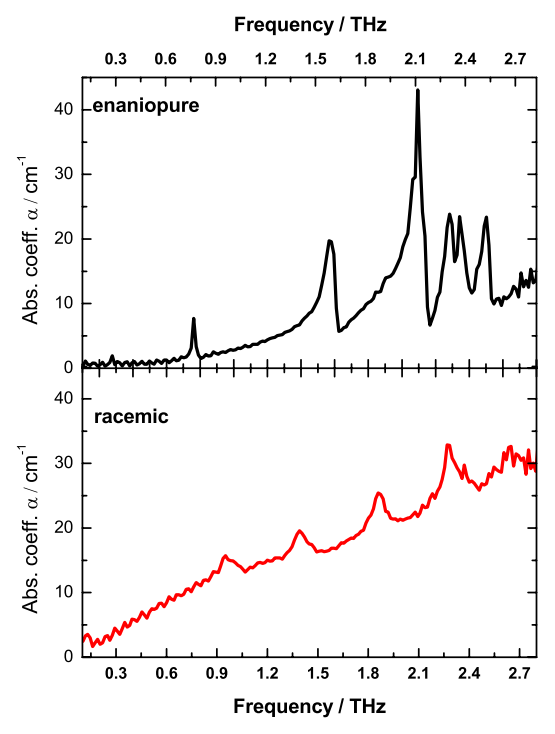

(a) Cystine

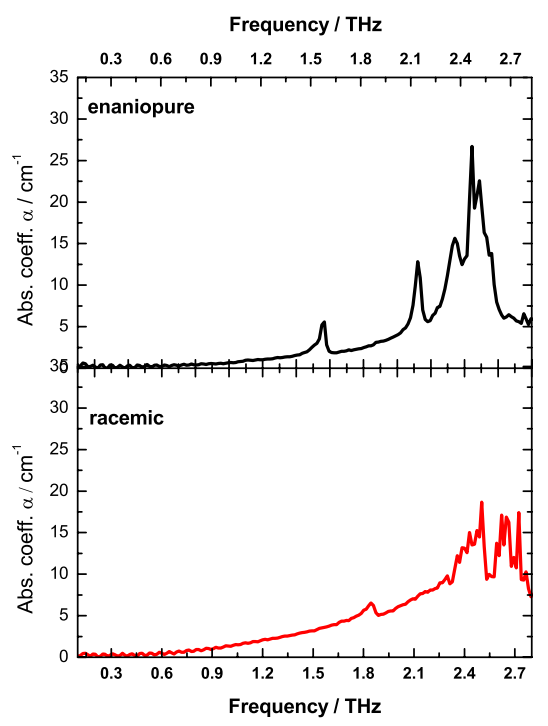

(c) Arabinose

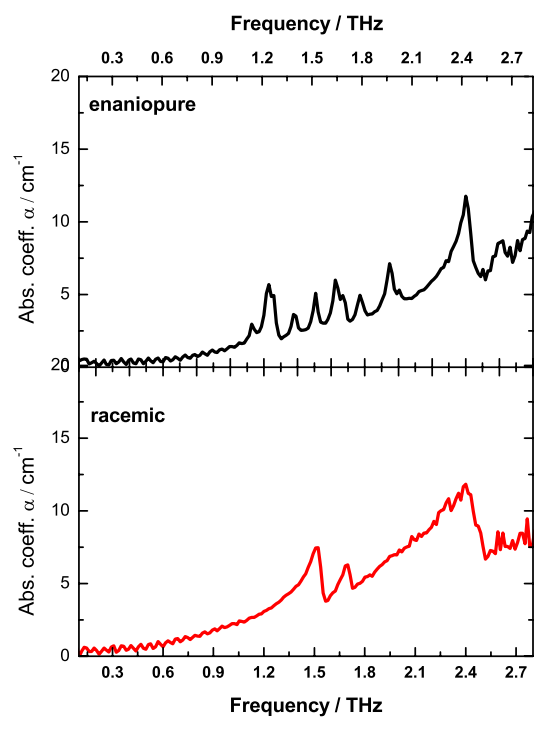

(b) Phenylactic Acid

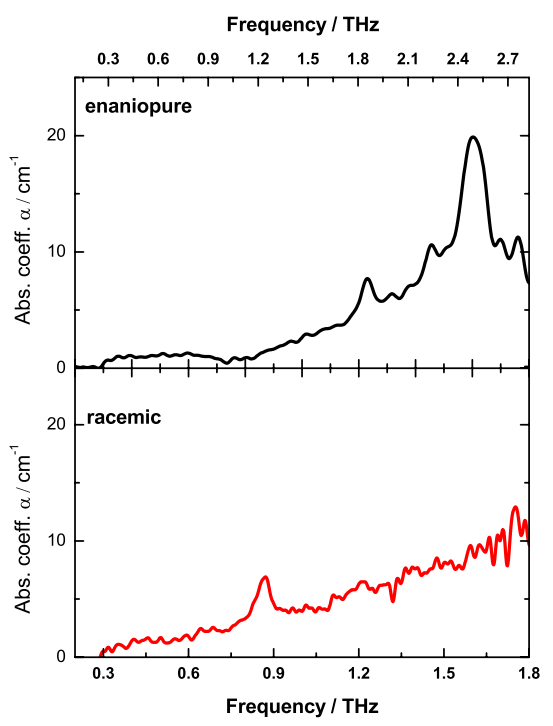

(d) Valine

Figure 3. Absorption spectra of four different enantiopure samples compared to the absorption spectra of the corresponding racemic compounds. The spectra (a), (b), (c) were recorded at a sample temperature of $15 \mathrm{~K}$, spectra (d) at $180 \mathrm{~K}$. The spectral features of the enantiopure samples differ significantly from those of their racemic counterparts. This clearly indicates the intermolecular character of the corresponding molecular vibrations.

enantiomers ( e.g. (+) Valine clockwise rotation of polarization vector \& (-) Valine anticlockwise rotation), or on the actual geometry of each enantiomer (e.g. D Valine \& L Valine, with the version synthesized from naturally occurring (+) Valine being considered the D form).

Enantiopure crystals are grown from a supersaturated solution containing only one of the enantiomers, thus the crystal contains only one type of molecule in a well ordered lattice, bound together by hydrogen bridges. In 
the case of racemic compounds one uses an equimolar solution of both of the enantiomers. The polarization of a light beam passing through such a solution will experience no change and such a crystal is named e.g. (+-) Valine or DL Valine.

Thus a crystal grown from a racemic solution will contain an equal amount of both of the enantiomers in a well ordered structure. The first of the two dimensional layers contains only one kind of molecule (e.g. D-Valine). The constituents of the crystal lattice are tied together by hydrogen bridges between the single molecules. The second layer is equal to the first one, however, it contains only molecules of the other type (e.g. L Valine), making it the mirror image of the first layer. These two layers are linked together in an alternating D, L, D, L pattern by hydrogen bridges themselves, making the racemic compound a hydrogen bonded network containing an equal amount of both enantiomers (e.g. DL Valine).

As the enantiopure crystals are just mirror images of each other, the terahertz spectra of the polycrystalline D- or L- Enantiomer are identical. In particular, the THz spectra of the racemic mixture - a mixture of polycrystalline pure Enantiomers - is identical to the spectrum of each of the enantiopure samples. In contrast, the far-infrared spectrum of the polycrystalline racemic compound differs significantly from the spectrum of the racemic mixture, although both samples consist of the same molecules. In Figure 3, the THz absorption spectra of four different enantiopure and racemic crystals are shown. The spectra of Cystine, Phenylactic Acid, and Arabinose were recorded at Freiburg/Germany using the transmission spectrometer described in Section 2. The spectra of the enantiopure samples shown in Figure 3 were recorded on the L-Enantiomer, however we also recorded the spectra of the D-Enantiomer as well as the racemic mixture which proved to be identical to the spectrum of the L-Enantiomer. In order to better resolve the spectroscopic features the samples were cooled to $15 \mathrm{~K}$. The spectrum of enantiopure and racemic Valine were recorded at Adelaide/Australia using the Picometrix T-ray 2000 spectrometer. Note the smaller bandwidth compared to the other three spectra. The samples were cooled to $180 \mathrm{~K}$ using a custom built liquid nitrogen cryocooler.

In all four cases the spectra of the racemic compound differ significantly from the spectra of the enantiopure samples, although they consist of the same molecules. The only differences are the intermolecular bonds between these molecules and their spatial arrangement relative to each other. Once more this clearly indicates the intermolecular character of the low-frequency vibrations that give rise to the characteristic fingerprints. We want to point out that $\mathrm{THz}$ spectroscopy is thus well suited to differentiate between enantiopure and racemic samples.

Interestingly, in all these cases shown here, the lowest mode observed in the racemic compound is found at higher frequencies compared to the lowest mode observed in the spectrum of the enantiopure sample. This could be explained - using the very basic model of a classical oscillator - by the denser packing and thus slightly stronger intermolecular bonds of the enantiomers in the crystalline structure of the racemic compound. This highlights the potential of $\mathrm{THz}$ spectroscopy to study the weak intermolecular binding forces in crystalline structures and will be subject of another publication.

\subsection{Single crystalline structures}

Most of the $\mathrm{THz}$ spectra of biomolecules reported in literature were obtained on polycrystalline samples, fine powders with an average grain size typically some tenths of a $\mu \mathrm{m}$ to avoid scattering. Due to the random distribution of the small crystalline clusters one does not expect any change of the spectra with respect to the polarization of the incident THz-radiation, depending on the sample orientation. This, however, changes if the sample consists of a large uniform single crystal. Now the molecules are all oriented in a well defined manner, which implies that only those IR-active vibrations that have at least in one component in the direction of the polarized $\mathrm{THz}$ radiation can be stimulated. In the case of sucrose it is relatively easy to obtain single crystalline samples. Sucrose crystallizes in the monoclinic system, ${ }^{15}$ space group P2 ${ }^{1}$ and the monoclinic angle is $b=102,983^{\circ}$. Such single crystals are sold as 'rock sugar' in candy shops, however care has to be taken to choose a pure monocrystal as most crystals show impurities such as needle-crystals, twins or conglomerates. A typical sucrose crystal is shown in Fig. (4), indicating the directions of the axes of the unit cell. The crystals were cut to obtain thin slices of 0.5 to $0.8 \mathrm{~mm}$ in thickness. 


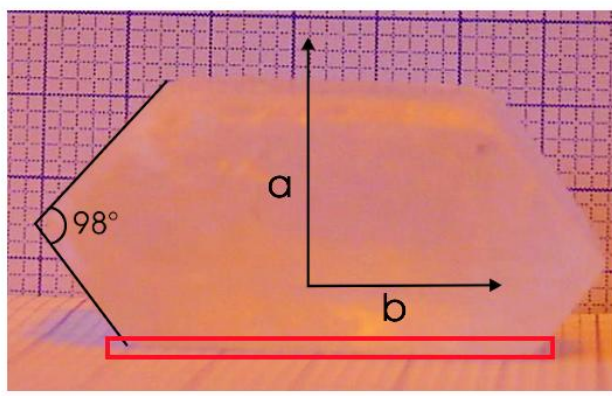

(a)

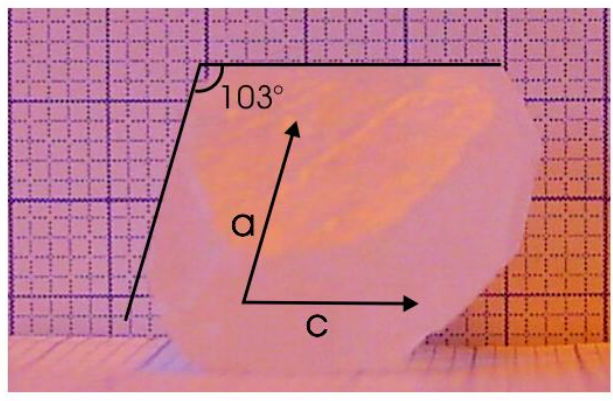

(b)

Figure 4. Sucrose crystal. The directions of the crystal axes are indicated together with some typical angles. A thin slice was cut from the crystal perpendicular to the paper plane in (a) as indicated by the red rectangular box.

Unfortunately the cutting of this sugar crystal is not as trivial as it may seem at first glance: The crystal is relatively unstable, and tends to break upon the slightest pressure. On the other hand, due to the relatively low melting point, good quality saws can easily get soiled and water cooling can obviously not be applied. Therefore in this work we present the spectra obtained with slices cut in the plane spanned by the $b$ - and $c$-axis, perpendicular to the paper plane in Fig. (4), as indicated by the rectangular box. Measurements in reflection geometry using the reflection spectrometer, presented in Section 2, avoids the problem of cutting the sample to thin slices.

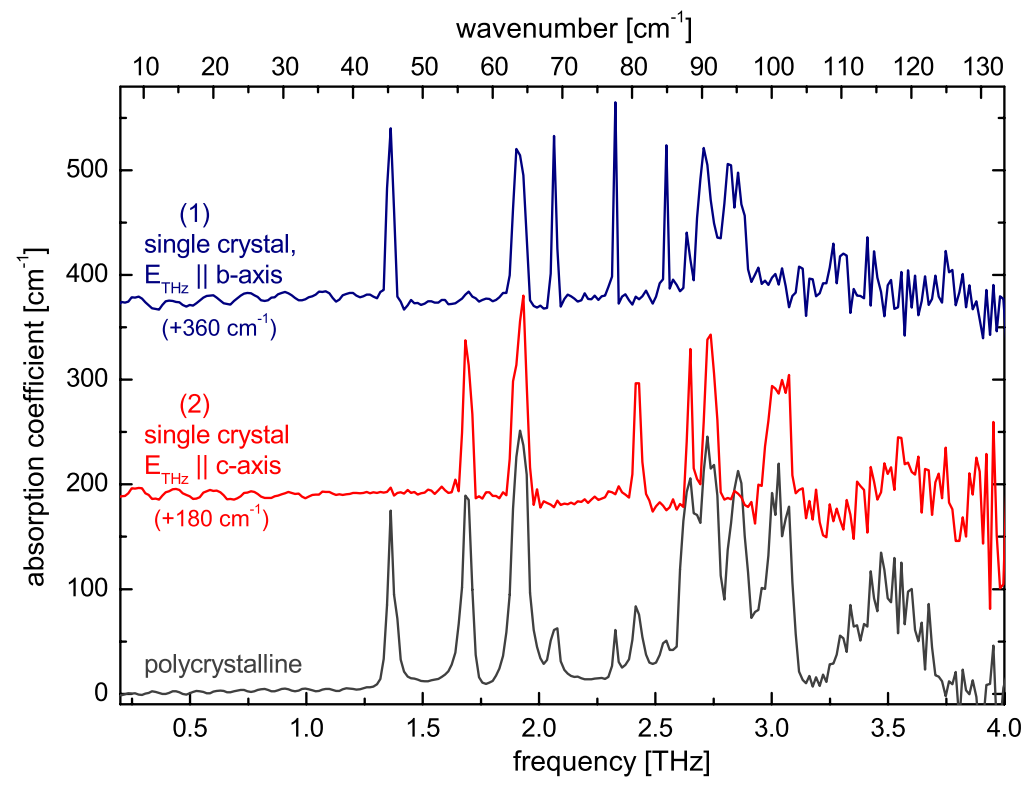

Figure 5. Polarization sensitive spectra of monocrystalline sucrose. The sample was orientated with its (1) $b$-axis respectively (2) c-axis parallel to the polarization of the incident THz-radiation. The lower curve shows the spectrum of polycrystalline sucrose. 
We recorded the spectra of the sample, with different orientations relative to the polarization of the incident $\mathrm{THz}$ radiation*. In Fig. (5) we present the spectra recorded when the polarization of the $\mathrm{THz}$ radiation was parallel to the $b$-axis (1) and the $c$-axis (2), respectively, of the sucrose monocrystal. The two spectra differ significantly from each other, however all the modes apparent in both spectra can be found, though with different intensities, in the spectrum of the polycrystalline sucrose. Some of the modes, however, are only visible if the monocrystalline sample is orientated in one direction. If we consider only the frequency range from 1 to 2 $\mathrm{THz}$, three modes are observed in the spectrum of the polycrystalline sample. The first, lowest-lying mode in the polycrystalline spectrum, is also clearly visible in spectrum (1). This mode is not at all stimulated if the sample is orientated with its c-axis parallel to the THz-field, indicating that the corresponding vibration has no IR-active component in the $c$-direction. The second mode of the polycrystalline sample at $\sim 55 \mathrm{~cm}^{-1}$ is strong and obvious in spectrum (2), in spectrum (1), however, only a weak mode is found at this frequency, indicating that the corresponding vibration is mainly localized along the $c$-axis but has also weak component along the $b$-axis. The third mode is strong and visible in both directions, with similar intensities, indicating that the components of this vibrations are equally distributed in both directions.

When we now consider the following three modes appearing in the spectrum of the polycrystalline sample, located in the range between $2 \mathrm{THz}$ and $2.5 \mathrm{THz}$, these modes are again solely found in either spectrum (1) or (2). However, although these modes are among the strongest in their respective spectra of the monocrystalline sample, they are relatively weak in the spectrum of the polycrystalline sucrose. Yet the shape, especially of the sharp mode at $77 \mathrm{~cm}^{-1}$, is as narrow in the spectrum of the polycrystalline sample as in spectrum (1). Therefore we assume that this mode does most probably not have a further component along the $a$-axis either, since it would have increased the intensity in the polycrystalline sample, indicating that the corresponding vibration can mainly be localized along the $b$-axis.

Finally we wish to point out another advantage of carrying spectroscopy on monocrystalline samples. Due to the homogeneity of the sample, no scattering occurs. Although scattering can also be minimized in polycrystalline samples by careful grinding of the powdery sample material, in those cases in which the sample cannot be carefully prepared for spectroscopy the scattering can have a non-negligible influence on the quality of the spectra.

\section{PROBING CRYSTALLINITY}

Having shown the high sensitivity of the $\mathrm{THz}$ spectra on different crystalline structures, the question arises whether $\mathrm{THz}$ spectroscopy could be a useful tool to probe crystallinity. In the previous section we have shown that the $\mathrm{THz}$ spectra can be used to distinguish between enantiopure and racemic crystals. In this section we wan to investigate the impact of a phase transition on the spectroscopic signatures in the far-infrared.

\subsection{Chlorobenzene}

Chlorobenzene is a toxic aromatic compound, with the chemical formula $\mathrm{C}_{6} \mathrm{H}_{5} \mathrm{C}_{1}$. At ambient temperature, the sample is a colorless liquid. Therefore, the sample was prepared for spectroscopy by filling the liquid sample in a cell covered by TPX windows separated by a metal spacer of $500 \mu \mathrm{m}$ thickness. To exclude any uncertainties arising from the sample thickness in the cell, similar measurements at room temperature - yet with significantly lower bandwidth - were performed on a $1 \mathrm{~mm}$ thick sample as well as in reflection geometry. In order to be able to record the temperature dependence of the terahertz spectra, the cell was mounted in the cryostat as described in Section 2.

The terahertz absorption spectra are shown in the upper graph in Figure 6. For a better representation, the individual traces recorded at different temperatures are shifted vertically by the amount given in the graph. It can be clearly seen that at temperatures above the melting point at $-45^{\circ} \mathrm{C}$ only a broad, featureless absorption is observed. However, when cooled down to temperatures below the melting point, the sample freezes out to a

\footnotetext{
${ }^{*}$ Typically the emission from $\mathrm{THz}$ antennas is described using the approximation of an ideal dipole, ${ }^{16}$ producing linearly polarized radiation.
} 


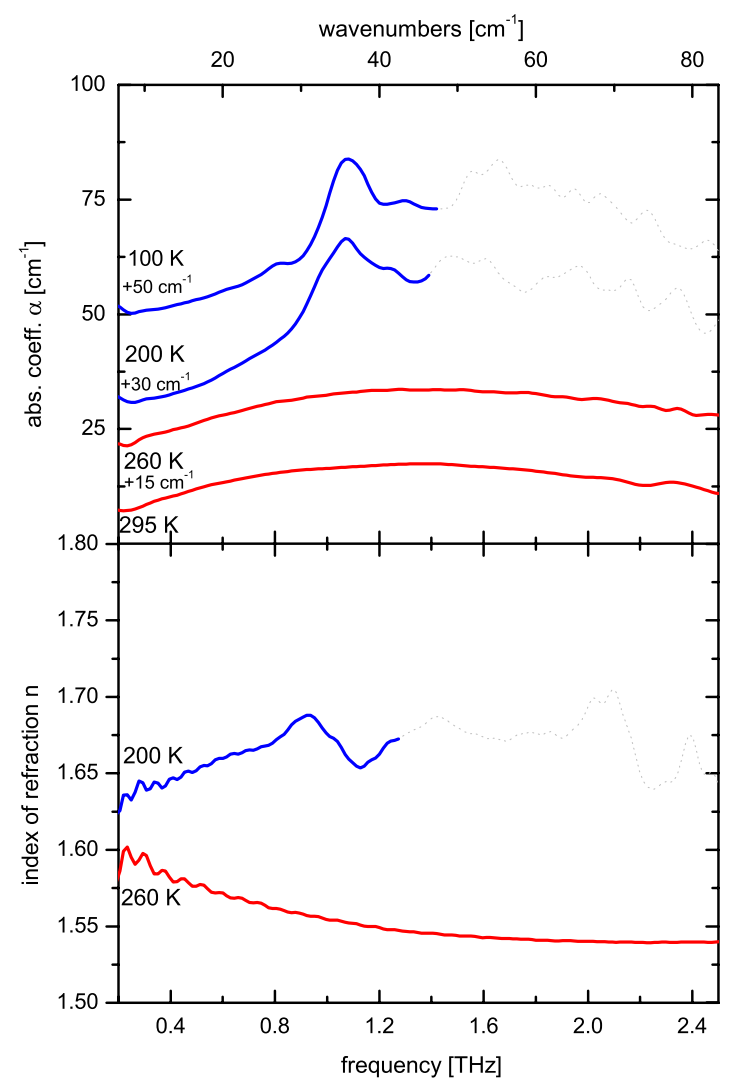

Figure 6. Temperature dependence of the absorption coefficient $\alpha$ and index of refraction of chlorobenzene. For better visibility the individual traces for the absorption coefficient are shifted vertically by an offset indicated in the figure. The index of refraction is shown representatively for two temperatures. Clear differences in the spectra of the liquid and polycrystalline samples are observed.

polycrystalline constitution, and sharp distinct absorption peaks arise in its far-infrared spectrum. Analogously to the absorption features observed in crystalline samples, upon further decrease of the temperature these peaks sharpen and shift slightly towards higher frequencies, as expected for lattice bands. The index of refraction, shown in the lower graph of Figure 6, shows the same behavior. At temperatures below the melting point, sharp resonance features appear in contrast to the featureless traces of the liquid sample. Furthermore, a clear difference in the general shape of the refraction spectrum is observed.

\subsection{PTFE and PCTFE}

In the previous example, the distinct absorption features arising from intermolecular vibrations could be used to probe the aggregation state of the sample. Some materials however also have a phase transition that is not correlated to a change of the aggregation state of the sample. This is quite often the case in polymers. Polytetrafluoroethylene (PTFE, better known under its brand name Teflon) is a long chain molecule that can be regarded as a PE in which all hydrogens are replaced by fluorine atoms. The solid-state phases of PTFE differ from those of $\mathrm{PE}$ in so far as the molecule is no longer a planar zig-zag chain, but is twisted into a helix. Therefore, PTFE has significantly more intermolecular bonds, and also the intermolecular forces are undoubtedly different from those in PE. Note that PTFE is therefore referred to as high-crystalline polymer. 


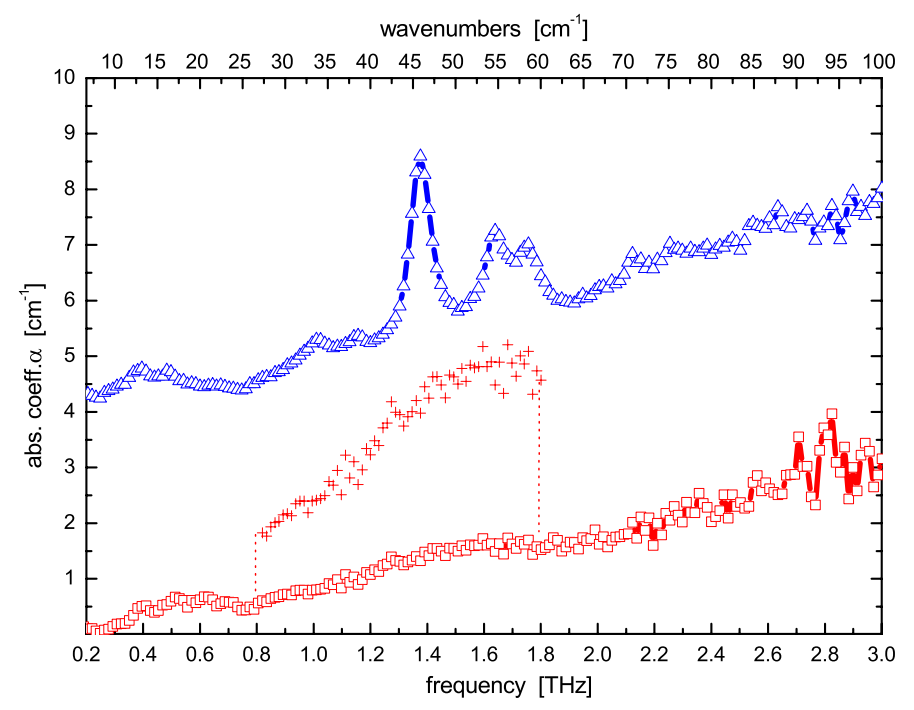

Figure 7. Absorption spectrum of PTFE at room temperature (and for better visibility magnified by a factor of 3 ) and cooled to $13 \mathrm{~K}$ (upper curve, shifted for better visibility by $4 \mathrm{~cm}-1$ ). Due to formation of crystalline regions in the polymer's low temperature solid phase constitution the relatively weak broad band at ambient temperature strengthens and splits into several sharp lines when cooled down to $10 \mathrm{~K}$.

Due to this crystalline character, the far-infrared spectrum of PTFE, shown in Figure (7), shows at $13 \mathrm{~K}$ several sharp resonances below $70 \mathrm{~cm}^{-1}$. At higher temperatures, i.e. above the solid-phase transition in PE, these bands merge into a broad weak band with the maximum near $50 \mathrm{~cm}^{-1}$. For better visibility, the absorption spectrum recorded at room temperature has been magnified by a factor of 3 in Fig. (7). Analogous to the resonances in PE in this frequency range, these absorptions in PTFE have been assigned by Bershtein et al ${ }^{17}$ to be liquid-lattice vibrations. Its splitting into series of bands at low temperatures occurs because the oscillations in the crystalline regions of polymer become lattice modes. ${ }^{17}$ The quite obvious difference between the absorption spectra at different temperatures, again, clearly demonstrates the potential of $\mathrm{THz}$ spectroscopy.

\section{CONCLUSION}

We have shown that many of the distinct, characteristic features dominating the $\mathrm{THz}$ spectra of most biomolecules originate from intramolecular vibrations rather than intermolecular vibrations. This high sensitivity on the intermolecular binding parameters can be used to distinguish between different racemic structures and might even be helpful in exploring the weak crystalline binding forces in these samples. Furthermore, THz spectroscopy can be used as a contact-free probe for detection of crystallinity of sealed samples.

\section{ACKNOWLEDGEMENT}

The authors would like to thank Hanspeter Helm and Peter Uhd Jepsen for fruitful discussions. We would further like to thank the BMBF, the EU project THz-BRIDGE under contract number QLK4- CT-2000-00129, the Australian Research Council and the Sir Ross and Sir Keith Smith foundation for financial support. 


\section{REFERENCES}

1. G. W. Chantry, Submillimetre Spectroscopy, Academic Press, London, 1971.

2. M. Hineno and H. Yoshinaga, "Far-infrared spectra of mono-, di - , and tri-saccharides in $50-16 \mathrm{~cm}^{-1}$ at liquid helium temperature," Spectrochimica Acta 30(A), pp. 411-416, 1973.

3. P. R. Smith, D. H. Auston, and M. Nuss, "Subpicosecond photoconducting dipole antennas," IEEE J. Quantum Electron. 24, pp. 255-260, 1988.

4. D. G. C. Fattinger, "Terahertz beams," Appl. Phys. Lett. 54, pp. 490-492, 1989.

5. A. G. Markelz, A. Roitberg, and E. J. Heilweil, "Pulsed terahertz spectroscopy of DNA, bovine serum albumin and collagen between 0.1 and 2.0 THz," Chem. Phys. Letters 320(1-2), pp. 42-48, 2000.

6. M. Walther, B. Fischer, M. Schall, H. Helm, and P. U. Jepsen, "Far-infrared vibrational spectra of all-trans, 9-cis and 13-cis retinal measured by THz time-domain spectroscopy," Chem. Phys. Lett. 332, pp. 289-295, 2000.

7. M. Walther, B. M. Fischer, and P. U. Jepsen, "Noncovalent intermolecular forces in polycrystalline and amorphous saccharides in the far infrared," Chemical Physics 288, pp. 261-268, 2003.

8. M. Walther, P. Plochocka, B. Fischer, H. Helm, and P. U. Jepsen, "Collective vibrational modes in biological molecules investigated by terahertz time-domain spectroscopy," Biopolymers (Biospectroscopy) 67, p. 310, 2002.

9. B. M. Fischer, H. Helm, and P. U. Jepsen, "T-ray spectroscopy of biomolecules: From chemical recognition towards biochip analysis - horizons and hurdles," Proceedings of SPIE 6038. article number 603809, 2006.

10. M. R. Kuttenruf, C. M. Brown, L. K. Iwaki, M. B. Capbell, T. M. Korter, and E. J. Heilweil, "Terahertz spectroscopy of short-chain polypeotides," Chemical Physics Letters 375, pp. 337-343, 2003.

11. B. Fischer, M. Hoffmann, H. Helm, G. Modjesch, and P. U. Jepsen, "Chemical recognition in terahertz time-domain spectroscopy and imaging," Semicond. Sci. Technol. 20, pp. S246-S253, 2005.

12. G.M.Day, J. A. Zeitler, W. Jones, T. Rades, and P. Taday, "Understanding the influence of polymorphism on phonon spectra: Lattice dynamics calculations and spectroscopy of carbamazepine," J. Phys. Chem. B 110, pp. 447-456, 2006.

13. P. U. Jepsen and B. M. Fischer, "Dynamic range in terahertz time-domain transmission and reflection spectroscopy," Optics Letters 30(1), pp. 29-31, 2005.

14. L. Thrane, R. H. Jacobsen, P. U. Jepsen, and S. R. Keiding, "THz reflection spectroscopy of liquid water," Chem. Phys. Lett. 240, p. 330, 1995.

15. C. Beevers, T. R. R. McDonald, J. Robertson, and F. Stern, "The crystal structure of sucrose," Acta Cryst. 5, pp. 689-690, 1951.

16. P. U. Jepsen, M. Schall, V. Schyja, C. Winnewisser, H. Helm, and S. R. Keiding, "Detection of high power thz pulses by phase retardation in an electro-optic crystal," in Ultrafast Processes in Spectroscopy, Svelto et al., eds., pp. 645-648, Plenum, New York, 1996.

17. V. A. Bershtein and V. A. Ryzhov, "Far infrared spectroscopy of polymers," Advances in Polymer Science 114, pp. 45-119, 1994. 